\title{
Determination of the Quasi-Geoid of Xinjiang and Tibet Areas and the Normal Height of Mt. Everest Based on EGM2008
}

\author{
WenBin Shen ${ }^{1,2, *}$, Jin $\mathrm{Li}^{1}$, Bin Tian ${ }^{3}$, and Jiancheng $\operatorname{Han}^{1}$ \\ ${ }^{I}$ Department of Geophysics, School of Geodesy and Geomatics, Wuhan University, Wuhan, China \\ ${ }^{2}$ Key laboratory of Geospace Environment and Geodesy, Wuhan University, Wuhan, China \\ ${ }^{3}$ Xinjiang Surveying Technique Center, Urumqi, China
}

Received 31 January 2010, accepted 26 July 2010

\begin{abstract}
The normal height $H^{*}$ is the height above the quasi-geoid, which coincides with the geoid in the ocean but slightly deviates from the geoid in terrestrial areas. In Mainland China, the height datum system is based on the quasi-geoid. The GPS technique provides the geodetic coordinates of an arbitrary point $P$ on the ground with an accuracy of better than $1 \mathrm{~cm}$. Hence, if the quasi-geoid is given, the normal height can subsequently be determined. In this study, we focus on the determination of the quasi-geoid of the Xinjiang and Tibet areas with a spatial resolution of $5^{\prime} \times 5^{\prime}$ as well as the normal height of Mt. Everest based on a newly released gravity field model EGM2008, which is complete to a degree and order of 2159. We determine the quasigeoid of Xinjiang and Tibet areas using the digital elevation model of the Shuttle Radar Topography Mission (SRTM), and determine the normal height of Mt. Everest using its geodetic coordinates provided by the Chinese State Bureau of Surveying and Mapping. Based upon the framework of Molodensky theory and EGM2008, we determined the $5^{\prime} \times 5^{\prime}$ quasi-geoid of the Xinjiang and Tibet areas and the normal height of Mt. Everest. Our results show that: (1) the accuracy of the determined $5^{\prime} \times 5^{\prime}$ quasi-geoid of Xinjiang and Tibet areas is around $0.2 \mathrm{~m}$ compared with 21 GPS leveling points distributed quite separately in the Xinjiang area (in the Tibet area the GPS/leveling data are not available except for the point at Mt. Everest); (2) the normal height of Mt. Everest is $8847.20 \mathrm{~m}$, which is very close to the value provided by the fourth geodetic surveying campaign on Mt. Everest by the Chinese State Bureau of Surveying and Mapping (the difference is $0.1 \mathrm{~m}$ ).
\end{abstract}

Key words: Normal height, Quasi-geoid of Xinjiang and Tibet areas, Mt. Everest, EGM2008

Citation: Shen, W. B., J. Li, B. Tian, and J. Han, 2011: Determination of the quasi-geoid of Xinjiang and Tibet areas and the normal height of Mt. Everest based on EGM2008. Terr. Atmos. Ocean. Sci., 22, 121-131, doi: 10.3319/TAO.2010.07.26.01(TibXS)

\section{INTRODUCTION}

A GPS surveying technique provides three dimensional (3-D) coordinates of a point on the ground with accuracy better than $1 \mathrm{~cm}$. Hence, using GPS technique can precisely determine the Cartesian coordinates $(x, y, z)$ or spherical coordinates $(r, \theta, \lambda)$ in the frame of geocentric reference, or the geodetic coordinates $(\varphi, \lambda, h)$ based on a reference ellipsoid (e.g., WGS84 ellipsoid). According to the GPS/leveling principle (Moritz 2000), the orthometric height of an arbitrary point $P$ can be determined by GPS, provided that the local geoid of the area including $P$ were given. However, in the areas where the local geoid is in poor accuracy or even no local geoid is available (e.g., Himalayan area

\footnotetext{
* Corresponding author

E-mail:wbshen@sgg.whu.edu.cn
}

or Antarctic area), with the geodetic coordinates of $P$, how to determine the orthometric height (or normal height) and geoid undulation (or height anomaly) using present available data becomes an interesting topic.

Based on the recently released $5^{\prime} \times 5^{\prime}$ global gravity model EGM2008 (Pavlis et al. 2008), one can determine the global quasi-geoid. Usually the Molodensky theory with zero-order approximation is used (Heiskanen and Moritz 1967), which may achieve sufficient precision in most areas on the Earth's surface, except for some plateaus, where higher order approximation should be applied. The quasigeoid coincides with the geoid in the ocean, and approximately coincides in plain areas, but deviates from the geoid by tens of centimeters to about 1 meter in highland areas. Since the normal height (above the quasi-geoid) deviates 
slightly from the orthometric height (above the geoid), it is practically useful and significant to determine the normal height in the areas where the orthometric height is difficult to access.

Precisely measuring the height of Mt. Everest has been one of focuses in geoscience since $19^{\text {th }}$ century. In 1852, a British team in India surveyed Mt. Everest using theodolites and declared that Mt. Everest was the highest peak on the Earth with the orthometric height of $8840 \mathrm{~m}$ (see e.g., Angus-Leppan 1982; Roy 1982). The orthometric height is the height above the geoid (Heiskanen and Moritz 1967), which is the equi-geopotential surface nearest to the mean sea level. In order to determine the orthometrc height of an arbitrary point $P$, traditionally we need a long-distance propagation by leveling and gravimetry, starting from a conventional (local) datum $D$ until to the interested point. In China, the datum $D$ is located in Qingdao (Qingdao Datum Origin). In the case where $P$ departs a long distance from $D$, the accumulated errors may be obvious. Hence, different results about the height of Mt. Everest are released in different times and by different institutions. In 1954, to further improve the measurement results provided by the British team in 1852, the Indian Surveying Bureau also measured the orthometric height of Mt. Everest and declared it being 8847.6 m (Gulatee 1954). In 1999, the United States" "Everest Surveying on the New Millennium" program suggested the orthometric height as $8850 \mathrm{~m}$ (Washburn 1999). In addition, organized by the Chinese State Bureau of Surveying and Mapping, the orthometric height of Mt. Everest was measured in 1975, 1998, and 2005, and was announced as $8848.13,8848.58$, and $8848.804 \mathrm{~m}$, respectively (Zhang et al. 2001; Chen et al. 2006; Yue 2006).

It is laborious to determine the orthomrtic height of Mt. Everest using traditional technology (including leveling and gravimetry). Theoretically, using the gravity-frequencyshift approach (Shen et al. 2008) one might directly and effectively determine the orthometric height, yet it may still take some time for this approach to be applied in practice due to the limited stability of present portable clocks.

This paper is organized as follows. We propose an approach, focusing on determining the normal height and height anomaly of an arbitrary point on the Earth's surface using the Molodensky theory with second-order approximation, based on the EGM2008 model. In section 3 we determine the $5^{\prime} \times 5^{\prime}$ quasi-geoid of Xinjiang and Tibet areas using the digital elevation model of the Shuttle Radar Topography Mission (SRTM), and in section 4 we determine the normal height of Mt. Everest using its geodetic coordi- nates. Finally, discussions and conclusions are provided in section 5 .

\section{METHOD}

\subsection{Calculation of the Potential at an Arbitrary Point $P$}

For a point $P$ on or above the Earth's surface, given its spherical coordinates $(r, \theta, \lambda)$, the gravitational potential at point $P$ could be determined by EGM2008 (Pavlis et al. 2008) based on spherical harmonic series expansion (Heiskanen and Moritz 1967; Chao et al. 2000):

$$
\begin{gathered}
V(r, \theta, \lambda)=\frac{G M}{r}\left[1+\sum_{n=2}^{N}\left(\frac{a}{r}\right)^{n} \sum_{m=0}^{n}\left(\bar{C}_{n m} \cos m \lambda+\bar{S}_{n m} \sin m \lambda\right)\right. \\
\left.\cdot \bar{P}_{n m}(\cos \theta)\right]
\end{gathered}
$$

where, the geocentric constant $G M$ equals $3986004.415 \mathrm{~m}^{3} \mathrm{~s}^{-2}$, $G$ and $M$ are the gravitational constant and the Earth's mass, respectively, $a$ is the Earth's equatorial average radius ( $a=$ $6378136.3 \mathrm{~m}), \bar{P}_{n m}$ are the full-normalized associated Legendre functions, $\bar{C}_{n m}$ and $\bar{S}_{n m}$ represent the spherical harmonic coefficients, and for the model EGM2008, the degree $n$ and order $m$ are complete to 2190 and 2159, respectively.

If the position of $P$ is provided as Cartesian coordinates $(x, y, z)$ or geodetic coordinates $(\varphi, \lambda, h)$, one can obtain its spherical coordinates (Moritz 1993), and then the gravitational potential can be determined by Eq. (1).

The geopotential $W(P)$ is the sum of the gravitational potential and the centrifugal force potential $Q(P)$ :

$W=V+Q \equiv V+\frac{1}{2} \omega^{2}\left(x^{2}+y^{2}\right) \equiv V+\frac{1}{2} \omega^{2} r^{2} \sin ^{2} \theta$

where $\omega$ is the spin rate of the Earth's rotation.

In our calculations, the WGS84 ellipsoid is adopted. The four fundamental parameters of the WGS84 ellipsoid are listed in Table 1 (NIMA 2000).

\subsection{Normal Height and Height Anomaly}

The disturbing potential at point $P$ is defined by the difference between the geopotential $W$ and the normal gravity potential $U$ :

$T=W-U$

where the normal gravity potential $U$ is determined based on

Table 1. Four fundamental parameters of the WGS84 ellipsoid.

\begin{tabular}{cccc}
\hline $\boldsymbol{a}(\mathbf{m})$ & $\boldsymbol{f}$ & $\boldsymbol{G M}\left(\mathbf{m}^{3} \mathbf{s}^{-2}\right)$ & $\boldsymbol{\Omega}\left(\mathbf{r a d ~ s}^{-1}\right)$ \\
\hline 6378137 & $1 / 298.257223563$ & $3986004.418 \times 10^{8}$ & $7292115 \times 10^{-11}$ \\
\hline
\end{tabular}


the four fundamental parameters as listed in Table 1. On the surface of the reference ellipsoid, the normal gravity potential holds the constant (Moritz 1993):

$\left.U\right|_{u=b}=U_{0}=\frac{G M}{E} \tan ^{-1} \frac{E}{b}+\frac{1}{3} \omega^{2} a^{2}$

where $U_{0}$ is equivalent to the geopotential constant $W_{0}$ on the geoid: $U_{0}=W_{0}$.

It should be pointed out that, in Molodensky theory (Molodensky et al. 1962; Moritz 1980), once given the position and geopotential at an arbitrary point on the ground surface of the Earth, the normal height $H^{*}$ can be determined (Heiskanen and Moritz 1967):

$$
H^{*}=\frac{C}{\bar{\gamma}}
$$

where $\bar{\gamma}$ is the average value of the normal gravity from $P$ to the corresponding point on the ellipsoidal surface along the normal gravity line, $C$ is the geopotential radix number of $P$ (Heiskanen and Moritz 1967), defined by

$C=W_{0}-W \equiv U_{0}-W$

With zero-order approximation, Eq. (5) is simplified as

$H_{(0)}^{*}=\frac{C}{\gamma_{0}}$

where $\gamma_{0}$ is the normal gravity at the corresponding point on the ellipsoidal surface, determined by the ellipsoid parameters.

In the areas where $P$ is located relatively high above the ellipsoidal surface (e.g., mountainous areas), higher order approximation is necessary. Generally, the secondorder approximation is accurate enough for the present accuracy requirement. The normal height with second-order approximation reads (Molodensky et al. 1962; Heiskanen and Moritz 1967; Moritz 1980):

$H_{(2)}^{*}=\frac{C}{\gamma_{0}}\left[1+\left(1+f+m-2 f \sin ^{2} \varphi\right) \frac{C}{\gamma_{0} a}+\left(\frac{C}{\gamma_{0} a}\right)^{2}\right]$

The height anomaly at $P$ is then determined by the famous Bruns formula:

$\zeta=\frac{T}{\gamma}$

where $\gamma$ is the normal gravity at the corresponding point $Q$ on the telluroid, a surface on which the normal gravity potential $U$ equals the geopotential $W$ at the corresponding point on the Earth's surface (e.g., Heiskanen and Moritz
1967).

With zero-order approximation the height anomaly is expressed as:

$\zeta_{(0)}=\frac{T}{\gamma_{0}}$

while the height anomaly with second-order approximation (Molodensky et al. 1962; Heiskanen and Moritz 1967; Moritz 1980) reads:

$\zeta_{(2)}=\frac{T}{\gamma_{0}} \cdot\left[1-2\left(1+f+m-2 f \sin ^{2} \varphi\right) \frac{H_{(2)}^{*}}{a}+3\left(\frac{H_{(2)}^{*}}{a}\right)^{2}\right]^{-1}$

Consequently, the quasi-geoid is determined once the height anomaly $\zeta$ is calculated at every point on the Earth's surface.

Neglecting the effect of the vertical deflection, the normal height $H^{*}$ and height anomaly $\zeta$ should satisfy the following relation:

$h=H^{*}+\zeta$

where $h$ is the geodetic height of point $P$. Equation (12) can be used to test the reliability of the results.

Equations (8) and (11) provide the normal height and height anomaly of an arbitrary point on the Earth's surface using Molodensky theory with second-order approximation.

\section{THE QUASI-GEOID OF XINJIANG AND TIBET AREAS}

To determine the quasi-geoid of an area, one needs the topographical information of the area to calculate the geopotential on the surface. We formulated the terrain of the Xinjiang and Tibet areas ranging from $25 \sim 50^{\circ} \mathrm{N}$ to $70 \sim 100^{\circ} \mathrm{E}$ by the digital elevation model (DEM) of SRTM which has a spatial resolution of 3" $\times 3$ " (about $90 \mathrm{~m}$ gridded) and an average vertical accuracy of about $16 \mathrm{~m}$ and with the EGM96 geoid as its vertical datum (Bamler 1999). We used version 4 of SRTM data in which the elevation values of the areas without original data are fitted by interpolation algorithms and auxiliary DEM data. Figure 1 shows the $5^{\prime} \times 5^{\prime}$ SRTM elevation (orthometric height above the EGM96 geoid) of the Xinjiang and Tibet areas. These results have been averaged based on the model SRTM with the spatial resolution $3 " \times 3 "$. The location of Mt. Everest is denoted by a star in Fig. 1.

The EGM96 geoid is released with the spatial resolution $15^{\prime} \times 15^{\prime}$ (Lemoine et al. 1998). We interpolated it in Xinjiang and Tibet areas by $5^{\prime} \times 5^{\prime}$ grids based on the cubic spline interpolation approach. The results are shown by Fig. 2 . With the gravitational potentials calculated based 


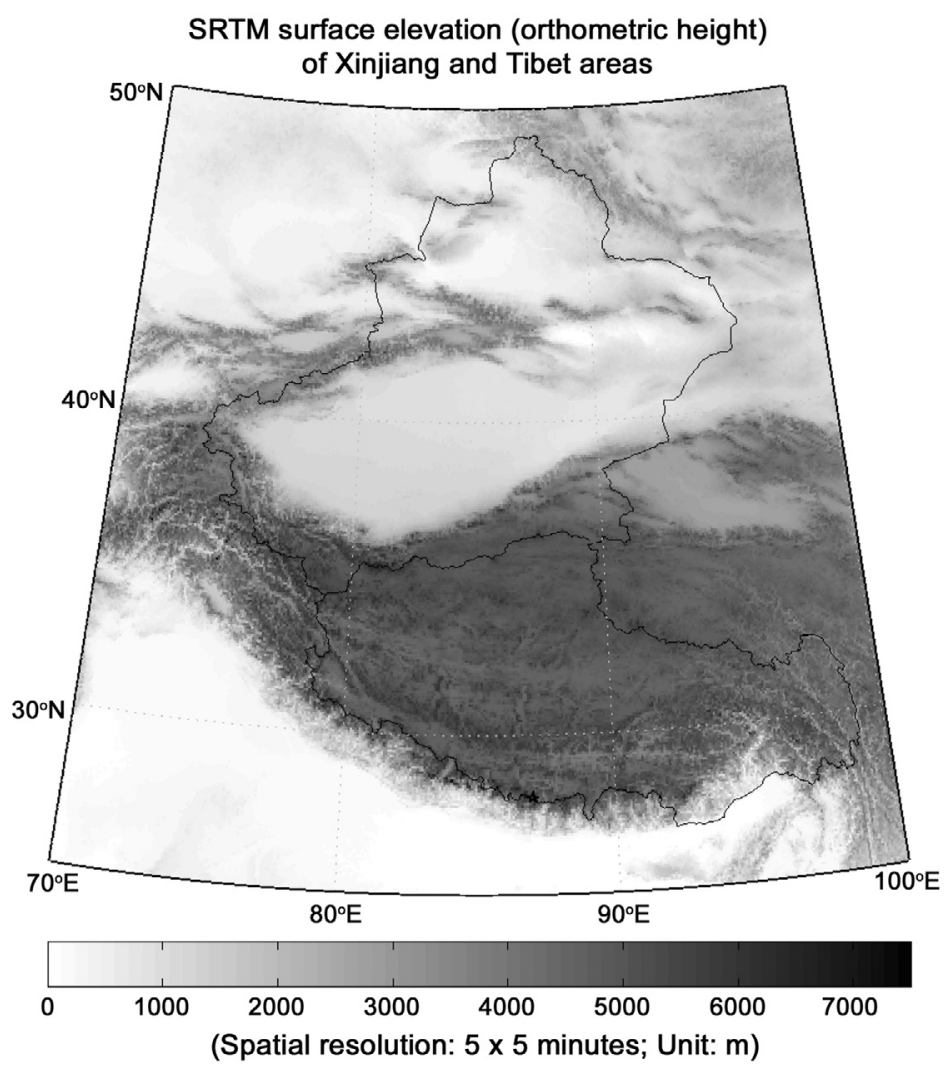

Fig. 1. SRTM elevation of Xinjiang and Tibet areas with $5^{\prime} \times 5^{\prime}$ resolution.

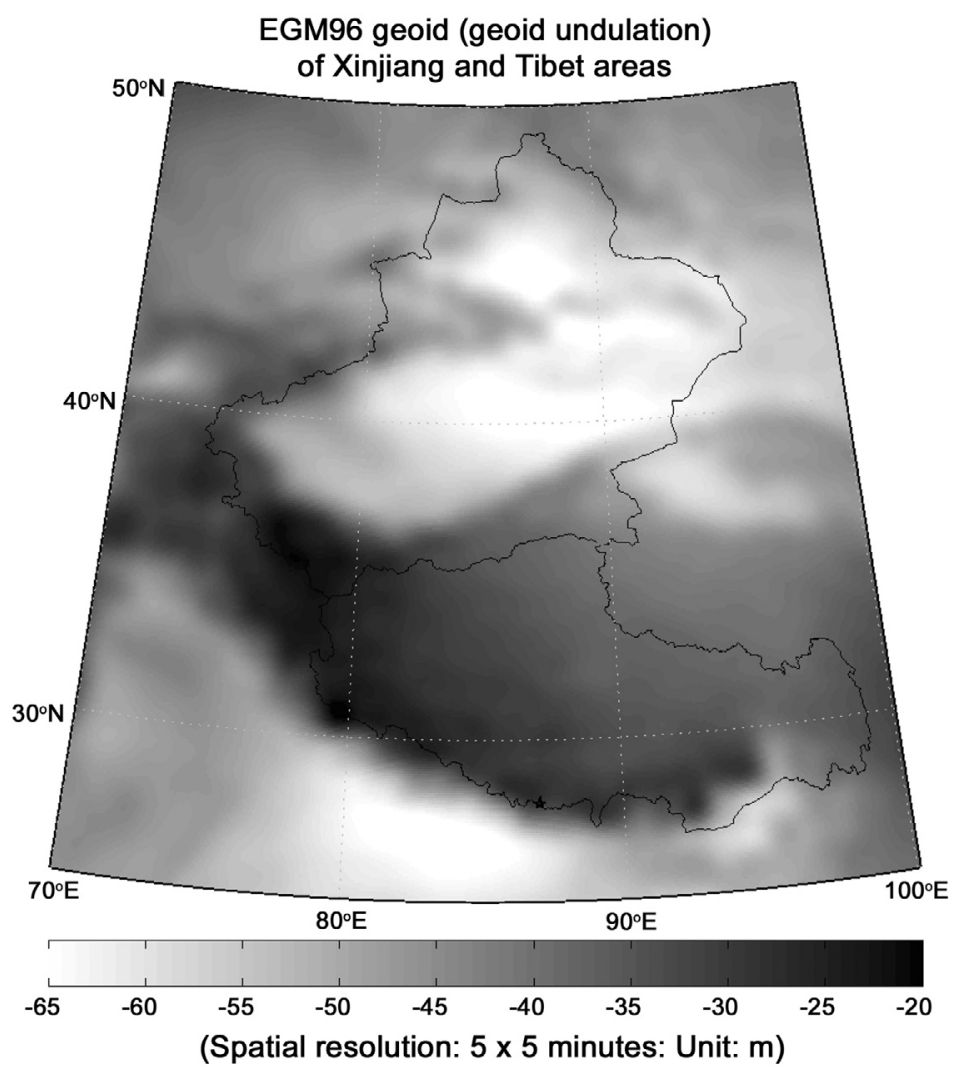

Fig. 2. EGM96 geoid of Xinjiang and Tibet areas with $5^{\prime} \times 5^{\prime}$ resolution based on cubic spline interpolation. 
on EGM2008 at the grid points on the geographical surface of Xinjiang and Tibet areas, we determined the $5^{\prime} \times 5^{\prime}$ grid height anomaly of the Xinjiang and Tibet areas based on Eqs. (1) - (12), using Molodensky theory with second-order approximation. The results are shown by Fig. 3 .

To evaluate the accuracy of the quasi-geoid of the Xinjiang and Tibet areas, the GPS/leveling data at 21 points (See Fig. 3 for their distribution) are used. The locations of the 21 testing points (note that we are only permitted to provide the approximate latitudes, longitudes and orthometric heights) and their comparative results between our calculations based on EGM2008 and the measured results at these testing points are listed in Table 2. The height anomalies at the testing points are calculated by cubic spline interpolation in the gridded quasi-geoid, which are then compared to the observed values provided by GPS/leveling. The statistical analysis results of the differences between our calculated results (based on EGM2008) and the measured ones at the $21 \mathrm{GPS} /$ leveling testing points are listed in Table 3.

From Table 2 we can see that, at 8 testing points (No. 1, $2,3,6,8,12,13$, and 18) the differences are around or less than $0.1 \mathrm{~m}$, and for the other points the differences range from 0.1 to $0.4 \mathrm{~m}$. The mean deviation of the calculated quasi-geoid is about $0.12 \mathrm{~m}$, with its RMS around $0.2 \mathrm{~m}$ (see Table 3).

\section{DETERMINATION OF THE NORMAL HEIGHT OF MT. EVEREST}

\subsection{The Gravitational Potential at Mt. Everest}

The geodetic coordinates of the snow peak at Mt. Everest were released by the Chinese State Bureau of Surveying and Mapping in the fourth geodetic surveying campaign on Mt. Everest as: $P$ (2759'17.09385', 8655'30.75851', $8821.094 \mathrm{~m})$, which are determined based on GPS observations (Zhang et al. 2001).

With the above geodetic coordinates and the four fundamental parameters of WGS84 ellipsoid (see Table 1), according to Eq. (1), the gravitational potential at Mt. Everest (snow peak) reads: $V_{P}=62465645.72 \mathrm{~m}^{2} \mathrm{~s}^{-2}$.

Since EGM2008 with degree 2190 has its spatial resolution of $5^{\prime} \times 5^{\prime}$, the gravitational potential derived from the above point-wise approach might be unreliable. Hence, an area-average approach was adopted: the gravitational potential was calculated from the average value based on the potentials at grid-points, in a selected area with some given width and its centre at the (snow) peak of Mt. Everest. The area selected is globally spherically gridded, with parallel latitude lines and longitude lines.

Setting the area $30^{\prime} \times 30^{\prime}($ about $50 \mathrm{~km} \times 50 \mathrm{~km})$ with interval of $0.2^{\prime}$ (about $400 \mathrm{~m}$ ), we have the gravitational

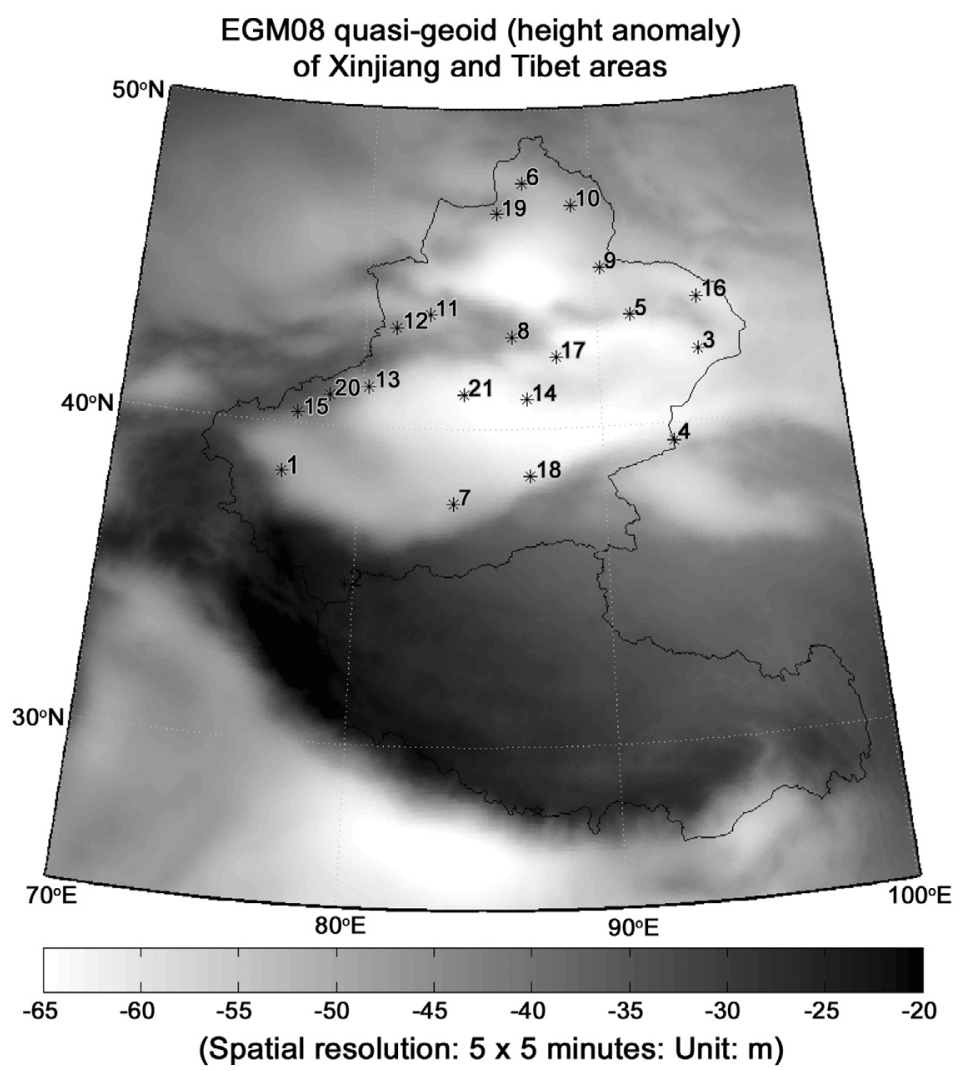

Fig. 3. 5' × 5' EGM2008 quasi-geoid of Xinjiang and Tibet areas. The $21 \mathrm{GPS} /$ leveling testing points (denoted by asterisk) are distributed in Xinjiang area. 
Table 2. Locations of the 21 GPS/leveling points and the differences between the calculated results based on EGM2008 and the measured results by GPS/leveling.

\begin{tabular}{|c|c|c|c|c|}
\hline $\begin{array}{c}\text { Site } \\
\text { Numbers }\end{array}$ & $\begin{array}{c}\text { Approximate } \\
\text { longitudes (d-m) }\end{array}$ & $\begin{array}{l}\text { Approximate } \\
\text { latitudes (d-m) }\end{array}$ & $\begin{array}{c}\text { Approximate } \\
\text { orthometric heights (m) }\end{array}$ & $\begin{array}{l}\text { Differences between } \\
\text { EGM2008 height anomaly } \\
\text { and GPS/leveling (m) }\end{array}$ \\
\hline 1 & 76.51 & 38.26 & 1211 & -0.0107 \\
\hline 2 & 79.41 & 35.01 & 4911 & -0.0650 \\
\hline 3 & 94.16 & 42.15 & 710 & 0.1056 \\
\hline 4 & 92.55 & 39.27 & 2126 & 0.1980 \\
\hline 5 & 91.26 & 43.28 & 811 & 0.2988 \\
\hline 6 & 86.51 & 47.41 & 429 & -0.0039 \\
\hline 7 & 83.52 & 37.39 & 1284 & -0.3198 \\
\hline 8 & 86.19 & 42.53 & 2091 & 0.0283 \\
\hline 9 & 90.14 & 44.59 & 924 & 0.2710 \\
\hline 10 & 89.05 & 46.59 & 792 & -0.2571 \\
\hline 11 & 82.46 & 43.34 & 803 & 0.1806 \\
\hline 12 & 81.20 & 43.08 & 1676 & 0.1069 \\
\hline 13 & 80.14 & 41.16 & 1129 & -0.0110 \\
\hline 14 & 86.55 & 40.55 & 802 & 0.3922 \\
\hline 15 & 77.19 & 40.19 & 1647 & 0.2762 \\
\hline 16 & 94.24 & 43.53 & 365 & 0.2950 \\
\hline 17 & 88.10 & 42.14 & 879 & 0.1720 \\
\hline 18 & 86.59 & 38.31 & 1036 & -0.0592 \\
\hline 19 & 85.42 & 46.44 & 1146 & 0.4128 \\
\hline 20 & 78.37 & 40.57 & 1854 & 0.2136 \\
\hline 21 & 84.14 & 41.04 & 867 & 0.2971 \\
\hline
\end{tabular}

Table 3. Statistical analysis of the residual errors of $21 \mathrm{GPS} /$ leveling testing points (unit: $\mathrm{m}$ ).

\begin{tabular}{cccc}
\hline Max & Min & Mean & RMS \\
\hline 0.4128 & -0.3198 & 0.1201 & 0.1978 \\
\hline
\end{tabular}

potential distribution as shown by Fig. 4. In Fig. 4, $\left(\phi_{0}, \lambda_{0}\right)$ are the latitude and longitude of the peak, and the average value of the potentials at the grid points in the area is expressed as $V_{\text {mean }}=62465632.84 \mathrm{~m}^{2} \mathrm{~s}^{-2}$.

The values of $V-V_{\text {mean }}$ in Fig. 4 increase with the decrease of the latitude, which implies that the value $V-V_{\text {mean }}$ is highly correlated with latitude. This attributes to the contribution of degree- 2 term of the gravitational field. We note that the gravitational potential values of the degree- 2 term increase from high to low latitude on a spherical surface, due to the oblateness of the Earth. After removing the degree- 2 term contributions, the residual potential distribution is shown in Fig. 5, from which one can see that the latitudinal correlation is significantly reduced. The amplitudes of Figs. 4 and 5 show that the degree- 2 term predominates over the potential distribution and suppresses the other parts of the field.

If we choose the $5^{\prime} \times 5^{\prime}$ area (about $10 \mathrm{~km} \times 10 \mathrm{~km}$ ) centered at Mt. Everest's peak and gridded by interval $0.05^{\prime}$ (about $100 \mathrm{~m}$ ), the model potential distribution is shown by Fig. 6 . If the $1^{\prime} \times 1^{\prime}$ area (about $2 \mathrm{~km} \times 2 \mathrm{~km}$ ) centered at Mt. Everest's peak and gridded by interval 0.01 ' (about $20 \mathrm{~m}$ ) is chosen, the model potential distribution is shown in Fig. 7.

As shown by Fig. 6 , the $5^{\prime} \times 5^{\prime}$ average value of the potential $V_{\text {mean }}$ equals $62465645.20 \mathrm{~m}^{2} \mathrm{~s}^{-2}$, while as shown by Fig. 7, the $1^{\prime} \times 1^{\prime}$ average value of that is $62465645.70 \mathrm{~m}^{2} \mathrm{~s}^{-2}$. 
These two values are quite close to the potential value given by the point-wise approach, due to the fact that the selected areas are not large. As the mean potential value with the area $1^{\prime} \times 1^{\prime}$ is closer to the potential value given by pointwise approach, we consider that the $1^{\prime} \times 1^{\prime}$ average value is a more appropriate choice.

Hence, by point-wise approach and 1 ' $\times 1$ ' area-average approach respectively, the gravitational model potential values at Mt. Everest read:

$$
\begin{aligned}
& V_{\text {point-wise }}=62465645.72 \mathrm{~m}^{2} \mathrm{~s}^{-2} \\
& V_{1^{\prime} \times 1^{\prime} \text { area-average }}=62465645.70 \mathrm{~m}^{2} \mathrm{~s}^{-2}
\end{aligned}
$$

\subsection{Normal Height and Height Anomaly of Mt. Everest}

The normal height and height anomaly of Mt. Everest can be determined by Eqs. (1) - (12), based on the gravitational model potential value at Mt. Everest. The results based on point-wise approach and 1' $\times 1$ ' area-average approach are listed in Table 4. For purposes of comparison, the results based on EGM96 using both point-wise approach and 1 ' $\times 1$ ' area-average approach are also listed in Table 4.

The calculations based on the gravity model EGM96 provide the potential value $62465634.13 \mathrm{~m}^{2} \mathrm{~s}^{-2}$ at the peak (by point-wise approach) and the average potential value $62465634.12 \mathrm{~m}^{2} \mathrm{~s}^{-2}$ of the $1^{\prime} \times 1^{\prime}$ area centered at the peak of

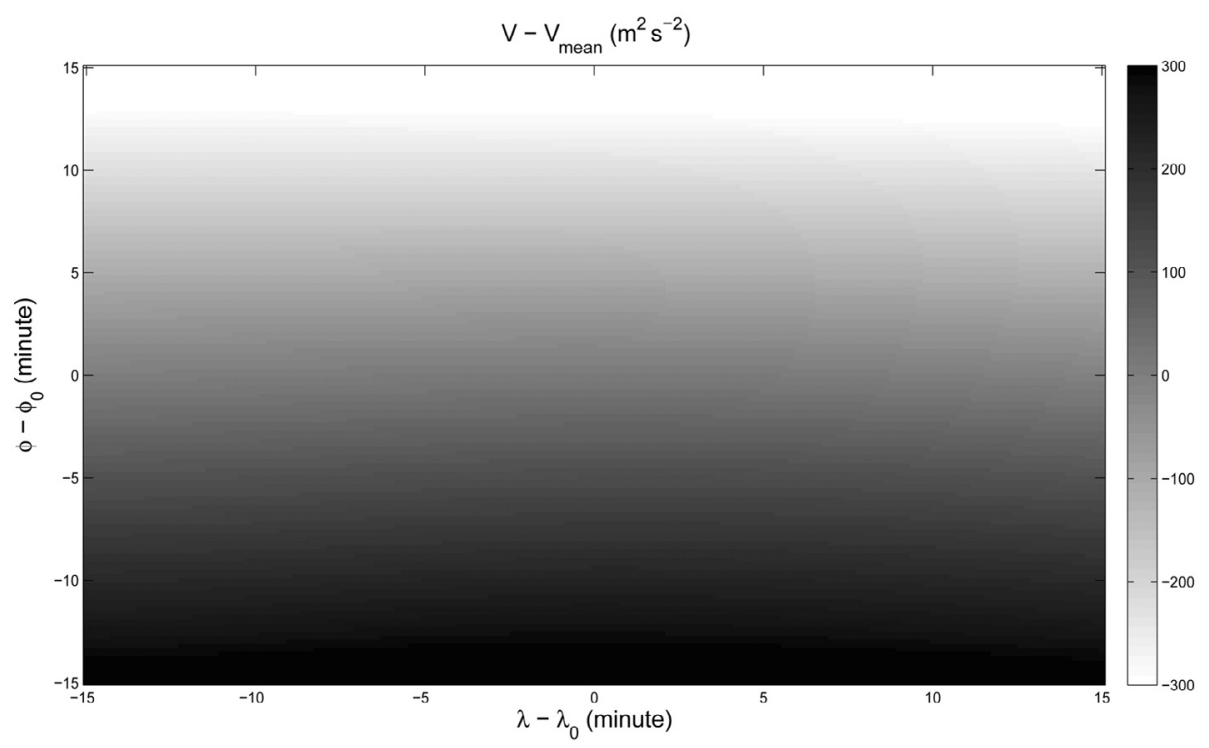

Fig. 4. EGM2008 model potential distribution of the $30^{\prime} \times 30^{\prime}$ area centered at Mt. Everest.

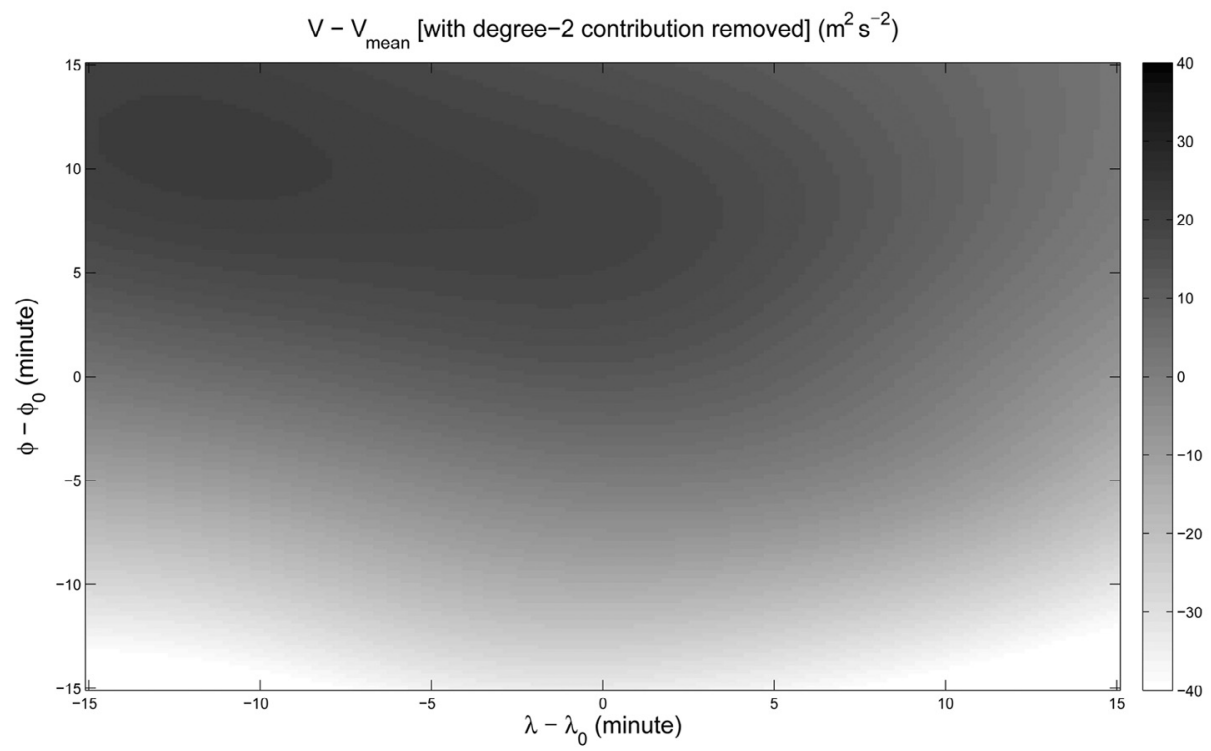

Fig. 5. EGM2008 model potential distribution of the 30' $\times 30^{\prime}$ area centered at Mt. Everest (in Fig. 1) without the degree- 2 term contributions. 


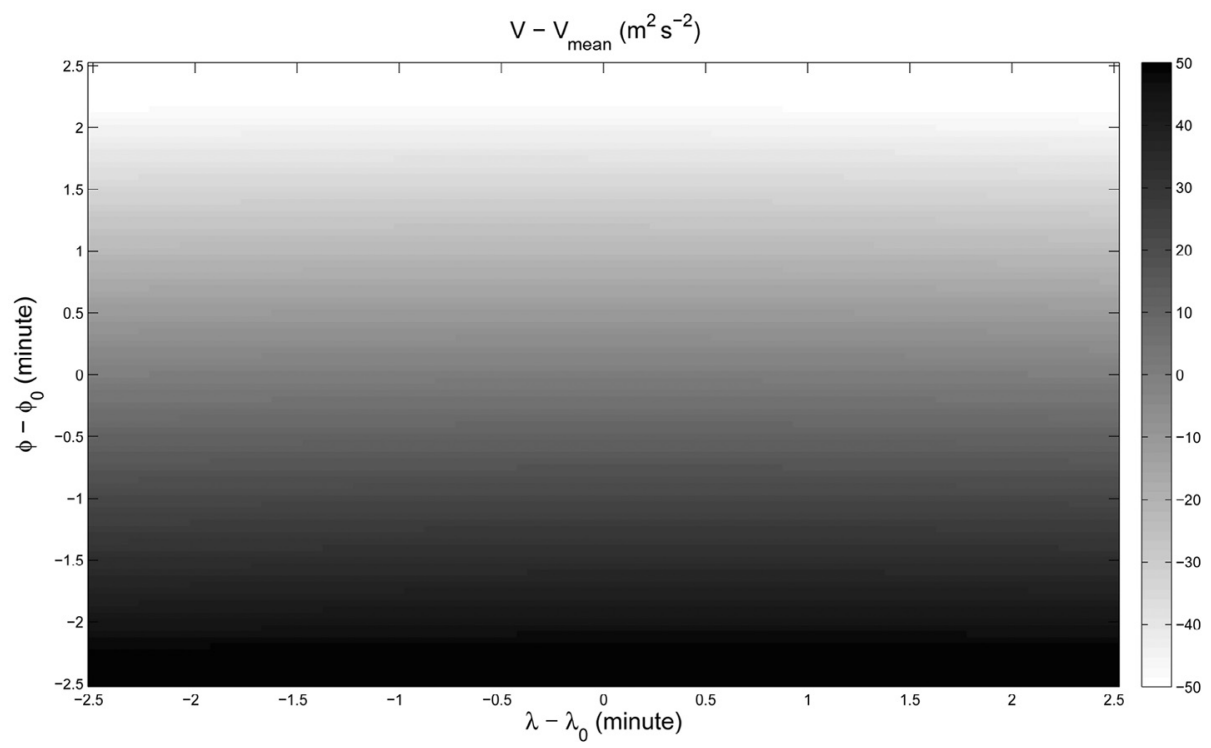

Fig. 6. EGM2008 model potential distribution of the $5^{\prime} \times 5^{\prime}$ area centered at Mt. Everest.

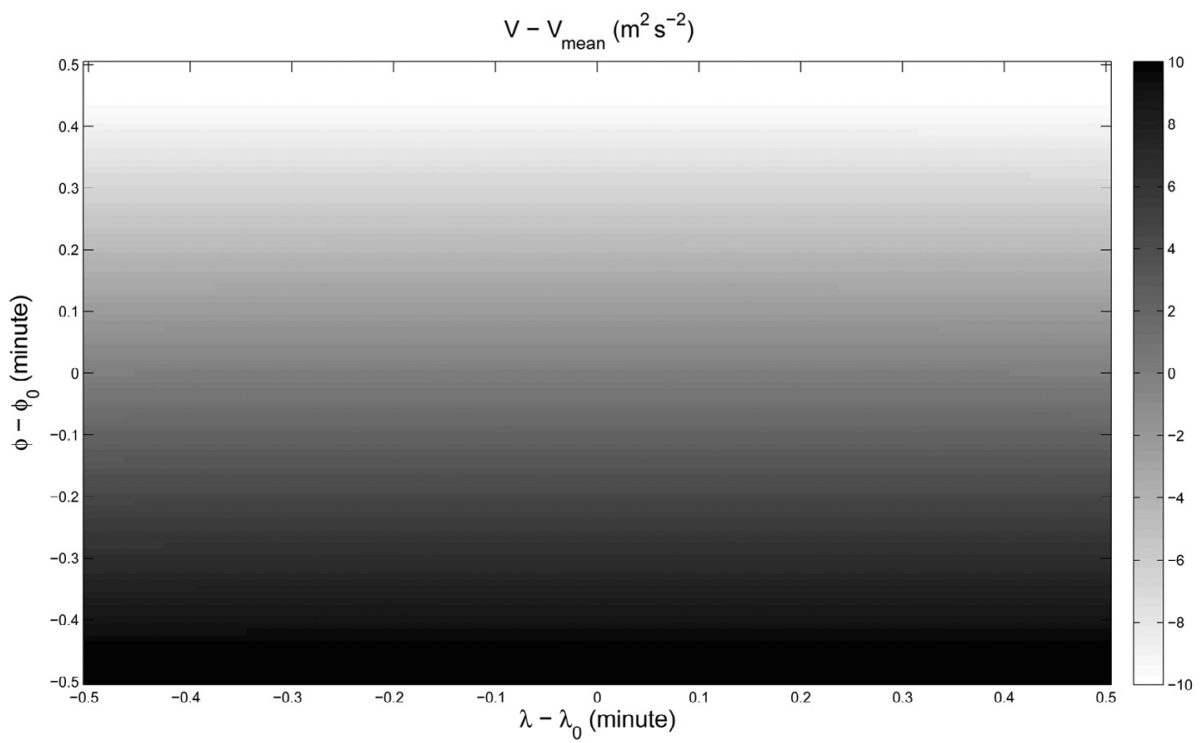

Fig. 7. EGM2008 model potential distribution of the $1^{\prime} \times 1^{\prime}$ area centered at Mt. Everest.

Table 4. Normal height and height anomaly of Mt. Everest determined based on EGM2008 and EGM96 (unit: m).

\begin{tabular}{|c|c|c|c|c|c|c|c|}
\hline \multirow{3}{*}{ Gravity model } & \multirow{3}{*}{ Approach } & \multicolumn{3}{|c|}{ Zero-order approximation } & \multicolumn{3}{|c|}{ Second-order approximation } \\
\hline & & Normal height & Height anomaly & Closed error & Normal height & Height anomaly & Closed error \\
\hline & & $\boldsymbol{H}_{(0)}^{*}$ & $\zeta_{(0)}$ & $\boldsymbol{H}_{(0)}^{*}+\zeta_{(0)}-\boldsymbol{h}$ & $\boldsymbol{H}_{(2)}^{*}$ & $\zeta_{(2)}$ & $\boldsymbol{H}_{(2)}^{*}+\zeta_{(2)}-\boldsymbol{h}$ \\
\hline \multirow{2}{*}{ EGM2008 } & Point-wise & 8834.87 & -26.02 & -12.25 & 8847.19 & -26.09 & -0.0007 \\
\hline & $1^{\prime} \times 1^{\prime}$ Area-average & 8834.88 & -26.03 & -12.25 & 8847.20 & -26.10 & -0.0007 \\
\hline \multirow{2}{*}{ EGM96 } & Point-wise & 8836.05 & -27.20 & -12.25 & 8848.37 & -27.28 & -0.0007 \\
\hline & $1^{\prime} \times 1^{\prime}$ Area-average & 8836.06 & -27.21 & -12.25 & 8848.38 & -27.29 & -0.0007 \\
\hline
\end{tabular}


Mt. Everest (by area-average approach). Based on the pointwise approach and area-average approach respectively, the potential differences between EGM2008 and EGM96 at the peak of Mt. Everest is 11.59 and $11.58 \mathrm{~m}^{2} \mathrm{~s}^{-2}$, which demonstrates that the height difference between the two international gravity models at Mt. Everest is about $1.18 \mathrm{~m}$.

From the closed errors in two cases (zero-order and second-order approximation, see Table 4), one could conclude that, in mountainous areas, to determine the normal height and height anomaly, the Molodensky approach with zero-order approximation could give rise to significant errors, while the Molodensky approach with second-order approximation may greatly improve the results.

\subsection{Accuracy Estimate of the Computed Normal Height}

The accuracy of the computed normal height of Mt. Everest depends on the model accuracy of the gravity field and the position accuracy of the interested point. The accuracy $\sigma_{h}$ of Mt. Everest's geodetic height given by Zhang et al. (2001) is $0.074 \mathrm{~m}$ [note that the geodetic height $h$ equals $8821.094 \pm 0.074 \mathrm{~m}$ as given by Zhang et al. (2001)].

The accuracy of the gravity field model depends on the commission accuracy and truncation accuracy. The commission accuracy is related to the accuracies $\left(\sigma_{\bar{C}_{n m}}\right.$ and $\left.\sigma_{\bar{S}_{m m}}\right)$ of the model coefficients as follows (Kaula 1966):

$\sigma_{c}=\frac{G M}{a}\left[\sum_{n=2}^{N} \sum_{m=0}^{n}\left(\sigma_{\bar{C}_{m m}}^{2}+\sigma_{\bar{S}_{m m}}^{2}\right)^{\frac{1}{2}}\right.$

And the truncation accuracy can be estimated by Kaula rule (Kaula 1966):

$\sigma_{t}=\frac{G M}{a}\left[\sum_{n=N+1}^{\infty} \sum_{m=0}^{n}\left(\sigma_{\bar{C}_{m n}}^{2}+\sigma_{\bar{S}_{m m}}^{2}\right)\right]^{\frac{1}{2}} \approx \frac{G M}{a} \cdot 10^{-5} \cdot\left[\sum_{n=N+1}^{\infty} \frac{2 n+1}{n^{4}}\right]^{\frac{1}{2}}$

Thus the model accuracy is determined by the error propagation law:

$\sigma_{\text {mod }}=\left(\sigma_{c}^{2}+\sigma_{t}^{2}\right)^{\frac{1}{2}}$

For the EGM2008 gravity model with $N=2190$, we have:

$\sigma_{c}^{E G M 08}=0.808 \mathrm{~m}^{2} \mathrm{~s}^{-2}, \sigma_{t}^{E G M 08}=0.286 \mathrm{~m}^{2} \mathrm{~s}^{-2}, \sigma_{\text {mod }}^{E G M 08}=0.86$ $\mathrm{m}^{2} \mathrm{~s}^{-2}$

While for the EGM96 gravity model with $N=360$, one gets:

$\sigma_{c}^{E G M 96}=3.527 \mathrm{~m}^{2} \mathrm{~s}^{-2}, \sigma_{t}^{E G M 96}=1.734 \mathrm{~m}^{2} \mathrm{~s}^{-2}, \sigma_{\text {mod }}^{E G M} 96=3.93$ $\mathrm{m}^{2} \mathrm{~s}^{-2}$
The accuracy of the height anomaly is due to the uncertainties of the geopotential model. Then the accuracy of the height anomaly reads:

$\sigma_{\zeta}=\frac{\sigma_{T}}{\gamma}$

Hence, we get:

$\sigma_{\zeta}^{E G M} 08=0.09 \mathrm{~m}, \sigma_{\zeta}^{E G M 96}=0.40 \mathrm{~m}$

The accuracy of the normal height could be estimated as

$\sigma_{H^{*}}=\left(\sigma_{h}^{2}+\sigma_{\zeta}^{2}\right)^{\frac{1}{2}}$

Thus, the accuracies of the determined normal height of Mt. Everest based on EGM2008 and EGM96 read respectively

$\sigma_{H}^{E G M 08}=0.12 \mathrm{~m}, \sigma_{H^{\prime}}^{E G M 96}=0.41 \mathrm{~m}$

\subsection{Comparisons with the Results Released by the Chi- nese State Bureau of Surveying and Mapping}

In the fourth geodetic surveying campaign on Mt. Everest by the Chinese State Bureau of Surveying and Mapping in 1998, by combining the GPS technique, leveling, gravimetry and other geodetic observations (Zhang et al. 2001), the normal height and height anomaly at Mt. Everest (snow peak) were determined with 8847.30 and $-26.08 \mathrm{~m}$, respectively. For purpose of comparison, our results and the results provided by the Chinese State Bureau of Surveying and Mapping in 1998 are listed in Table 5.

From Table 5 we can see that the difference between the normal height determined in this study (using area-average approach and based on EGM2008) and the normal height released by the Chinese State Bureau of Surveying and Mapping is $0.1 \mathrm{~m}$ (and the difference between the two height anomalies is less than $0.1 \mathrm{~m}$ ), while the difference between the normal height (height anomaly) based on EGM96 and that released by the Chinese State Bureau of Surveying and Mapping is larger than $1 \mathrm{~m}$. We note that the difference between the calculated normal height based on EGM2008 and the measured result agrees well with the estimated accuracy $0.12 \mathrm{~m}$ as given by Eq. (18). We also note that the local quasi-geoid at Mt. Everest determined by EGM96 locates about $1.2 \mathrm{~m}$ lower than that by EGM2008.

\section{DISCUSSIONS AND CONCLUSIONS}

Given the 3-D spatial coordinates (in Cartesian, spherical or geodetic form) of an arbitrary point on the surface of the Earth, one can determine its normal height and height anomaly using EGM2008 following the trace of Moloden- 
Table 5. Comparisons of the results of the normal height and height anomaly at Mt. Everest (snow peak) determined in this study and the Chinese State Bureau of Surveying and Mapping (unit: $\mathrm{m}$ ).

\begin{tabular}{|c|c|c|c|c|c|}
\hline \multirow{3}{*}{ Results } & \multicolumn{4}{|c|}{ Results of this study } & \multirow{3}{*}{$\begin{array}{l}\text { Results released by the } \\
\text { Chinese State Bureau } \\
\text { of Surveying and } \\
\text { Mapping in } 1998\end{array}$} \\
\hline & \multicolumn{2}{|c|}{ EGM96 } & \multicolumn{2}{|c|}{ EGM2008 } & \\
\hline & Point-wise & $\left(1^{\prime} \times 1^{\prime}\right)$ Area-average & Point-wise & $\left(1^{\prime} \times 1^{\prime}\right)$ Area-average & \\
\hline Normal height & $8848.37 \pm 0.41$ & $8848.38 \pm 0.41$ & $8847.19 \pm 0.12$ & $8847.20 \pm 0.12$ & 8847.30 \\
\hline Height anomaly & $-27.28 \pm 0.40$ & $-27.29 \pm 0.40$ & $-26.09 \pm 0.09$ & $-26.10 \pm 0.09$ & -26.08 \\
\hline
\end{tabular}

sky theory. The accuracies of the calculated normal height and height anomaly depend on the accuracies of the given coordinates and gravity model. Since the GPS technique provides the 3-D coordinates of a point with an accuracy higher than $1 \mathrm{~cm}$, the accuracy of the determined normal height provided in this study depends mainly on the accuracy of EGM2008.

Once the height anomaly of every point in an interesting area is calculated, the quasi-geoid of the area is determined. However, the determination of a local (or global) quasi-geoid needs coordinates of points distributed densely on the surface. Despite its high accuracy, the present GPS technique might not satisfy the "dense distribution" requirement. To determine the quasi-geoid of the Xinjiang and Tibet areas, we used the digital elevation model SRTM to formulate the terrain of the areas. Although the dense distribution of the grid points on the Earth's surface is ensured by its high spatial resolution, the accuracy of SRTM is obviously much lower than that provided by GPS observations. However, Li et al. (2009) has demonstrated by various experiments that the coordinate errors of the surface points are not sensitive in determining the local (or global) quasigeoid, and the deviation of the height anomaly may not exceed $1 \mathrm{~cm}$ in the case that the model SRTM is used to locate the Earth' surface. Hence, a local $5^{\prime} \times 5^{\prime}$ quasi-geoid of the Xinjiang and Tibet areas with relatively high accuracy is then properly determined based on EGM2008 and SRTM.

In this study, based on EGM2008 and the geodetic coordinates of the snow peak of Mt. Everest, we determined the normal height and height anomaly of Mt. Everest (snow peak). Our results coincide very well with the results released by the Chinese State Bureau of Surveying and Mapping in 1998 (the difference is $0.1 \mathrm{~m}$ ).

Pavlis et al. (2008) pointed out that the EGM2008 has a global accuracy of around $10 \mathrm{~cm}$ and with a spatial resolution of $5^{\prime} \times 5^{\prime}$. In one aspect, our results of the normal height of Mt. Everest based on EGM2008 agree well with the results released by the Chinese State Bureau of Surveying and Mapping (with difference of $0.1 \mathrm{~m}$ ), and in another aspect, by testing $21 \mathrm{GPS} /$ leveling points in Xinjiang area, we conclude that the average accuracy of the EGM2008 quasi-geoid of the Xinjiang and Tibet areas is about $0.2 \mathrm{~m}$ (with its mean systematic deviation around $0.1 \mathrm{~m}$ ). Taking into account that the Xinjiang and Tibet areas are plateau areas with relatively complicated geological structure, we may safely conclude that our results in the present study provide a good confirmation of the global 10-centimeterlevel accuracy of the EGM2008.

Finally, it is noted that, based on our calculations, the Molodensky approach with second-order approximation should be used in the determination of the normal height and height anomaly in highland areas.

Acknowledgements The authors are grateful to the two anonymous reviewers for their insightful comments and valuable suggestions, which greatly improved the manuscript. This study was supported by Natural Science Foundation of China (Grant No. 40974015 and 40637034), National 863 Project of China (Grant No. 2006AA12Z211), and the Fund of Key Laboratory of Geospace Environment and Geodesy, Ministry of Education, China (No. 07-12).

\section{REFERENCES}

Angus-Leppan, P. V., 1982: The Height of Mt. Everest. Surv. Rev., 26, 367-385.

Bamler, R., 1999: The SRTM mission: The SRTM Mission: A world-wide $30 \mathrm{~m}$ resolution DEM from SAR Interferometry in 11 days. Photogramm. Week, 99, 145-154

Chao, B. F., E. C. Pavlis, C. Hwang, C. C. Liu, C. K. Shum, C. L. Tseng, and M. Yang, 2000: COSMIC: Geodetic applications in improving Earth's gravity model. Terr. Atmos. Ocean. Sci., 11, 365-378.

Chen, J., J. Yuan, C. Guo, Y. Zhang, and P. Zhang, 2006: Progress in technology for the 2005 height determination of Qomolangma Feng (Mt. Everest). Sci. China Ser. D: Earth Sci., 49, 531-538, doi: 10.1007/s11430006-0531-1. [Link]

Gulatee, B. L., 1954: The height of Mt. Everest: A new determination (1952-1954). Technical Paper, No. 8, New Deli: Survey of India, 1-80.

Heiskanen, W. A. and H. Moritz, 1967: Physical Geodesy. W. H. Freeman \& Co. Ltd., San Francisco, 364 pp. 
Kaula, W. M., 1966: Theory of Satellite Geodesy. Blaisdell Publishing Co., Waltham, Massachusetts.

Lemoine, F. G., S. C. Kenyon, J. K. Factor, R. G. Trimmer, N. K. Pavlis, D. S. Chinn, C. M. Cox, S. M. Klosko, S. B. Luthcke, M. H. Torrence, Y. M. Wang, R. G. Williamson, E. C. Pavlis, R. H. Rapp, and T. R. Olson, 1998: The development of the joint NASA GSFC and the National Imagery and Mapping Agency (NIMA) geopotential model EGM96. Technical Report NASA Technical Paper NASA/TP-1998-206861, Goddard Space Flight Center, Greenbelt.

Li, J., W. B. Shen, and J. C. Han, 2009: Study on the determination of the normal height based on EGM2008 and 3-D coordinates. Symposium for Ph.D. Candidates in the Science of Surveying and Mapping, Zhenzhou, China, 2009-05, 59-65. (in Chinese)

Molodensky, M. S., V. F. Eremeev, and M. I. Yurkina, 1962: Methods for Study of the External Gravitational Field and Figure of the Earth. Israel Program for Scientific Translations (Jerusalem), 248 pp.

Moritz, H., 1980: Advanced Physical Geodesy. Wichmann, Karlsruhe.

Moritz, H., 1993: The Figure of the Earth. Wichmann, Karlsruhe.

Moritz, H., 2000: Molodensky's Theory and GPS. Mittelungen der geodätischen Institute der Technischen Universität Graz, Folge 88, Graz.

NIMA (National Imagery and Mapping Agency), 2000: De- partment of Defense World Geodetic System 1984: Its Definition and Relationships with Local Geodetic Systems. Third edition, National Imagery and Mapping Agency technical report, TR8350.2.

Pavlis, N. K., S. A. Holmes, S. C. Kenyon, and J. K. Factor, 2008: An Earth gravitational model to degree 2160: EGM2008. Presented at the 2008 General Assembly of the European Geosciences Union, Vienna, Austria, 2008, April, 13-18.

Roy, R. D., 1982: An outline survey of some aspects of technology in India. 1750-1900 and its legacy. Indian J. Hist. Sci., 17, 18-27.

Shen, W. B., J. Ning, J. Li, J. Liu, and D. Chao, 2008: The concept of direct orthometric height determination based on frequency shift equation. In: Li, D., J. Gong, and $\mathrm{H}$. Wu (Eds.), International Conference on Earth Observation Data Processing and Analysis (ICEODPA), Proc. SPIE, Vol. 7285, 728512, doi: 10.1117/12.816357. [Link]

Washburn, B., 1999: New Official Height of the Everest. National Geographic, $76 \mathrm{pp}$.

Yue, J. L., 2006: The key technique research and accuracy evaluation of Qomolangma's measurement. Ph.D. Thesis, Wuhan University, China. (in Chinese)

Zhang, Q. D., J. Y. Chen, S. Y. Pang, J. Zhang, and Z. M. Wang, 2001: The fourth geodetic surveying campaign on Mt. Everest and its adjacent area. Sci. Surv. Mapp., 26, 10-15. (in Chinese) 